\title{
Magnetic nanoparticles in cancer diagnosis, drug delivery and treatment (Review)
}

\author{
MEIJIA WU and SHENGWU HUANG \\ Department of Pharmacy, Zhejiang Chinese Medical University, Hangzhou, Zhejiang 311402, P.R. China
}

Received February 21, 2017; Accepted May 17, 2017

DOI: $10.3892 / \mathrm{mco} .2017 .1399$

\begin{abstract}
In recent years, magnetic nanoparticles (MNPs) have demonstrated marked progress in the field of oncology. General nanoparticles are widely used in tumor targeting, and the intrinsic magnetic property of MNPs makes them the most promising nanomaterial to be used as contrast agents for magnetic resonance imaging (MRI) and induced magnetic hyperthermia. The properties of MNPs are fully exploited when they are used as drug delivery agents, wherein drugs may be targeted to the desired specific location in vivo by application of an external magnetic field. Early diagnosis of cancer may be achieved by MRI, therefore, individualized treatment may be combined with MRI, so as to achieve the precise definition and appropriate treatment. In the present review, research on MNPs in cancer diagnosis, drug delivery and treatment has been summarized. Furthermore, the future perspectives and challenges of MNPs in the field of oncology are also discussed.
\end{abstract}

\section{Contents}

1. Introduction

2. Cancer diagnosis

3. Drug delivery

4. Cancer treatment

5. Conclusions and perspectives

\section{Introduction}

Magnetic nanoparticles (MNPs) are a kind of intelligent nanomagnetic material, with small particle size, large specific surface area, magnetic response and superparamagnetism (1). MNPs may be assembled and positioned under a constant magnetic field, and the heat is absorbed by the electromagnetic wave in the alternating magnetic field. In biomedical applications,

Correspondence to: Professor Shengwu Huang, Department of Pharmacy, Zhejiang Chinese Medical University, Gaoke Road, Hangzhou, Zhejiang 311402, P.R. China

E-mail: hsw55@163.com

Key words: cancer, magnetic nanoparticles, cancer diagnosis, drug delivery, treatment
MNPs are generally in the superparamagnetic state $(2,3)$. The most frequently used nanomaterial is the iron oxide nanoparticle, including magnetite $\left(\mathrm{Fe}_{3} \mathrm{O}_{4}\right)$ and maghemite $\left(\gamma-\mathrm{Fe}_{2} \mathrm{O}_{3}\right)$ (4). It is well known that MNPs have an important role in cancer diagnosis, drug delivery and treatment. For cancer diagnosis, tumor imaging technology opened the possibility of early detection of disease. Common imaging modalities include magnetic resonance imaging (MRI) (5), magneto acoustic tomography (MAT) (6), computed tomography (CT) (7) and near-infrared (NIR) imaging (8). Among them, MRI has a strong influence in the early diagnosis of cancer, and superparamagnetic iron oxide nanoparticles (SPIONs) are the most representative as a contrast agent for MRI (9). Currently, certain iron oxide-based MNPs have been approved for use in clinical MRI, for example ferumoxil (GastroMARK) enhances imaging of the bowel (10). Due to the small size and large specific surface area of MNPs, they are able to easily reach the location of the lesion (11). Therefore, MNPs as a drug carrier for drug delivery is an application that cannot be ignored. This property of MNPs is fully exploited when they are used as drug delivery agents, wherein drugs may be targeted to the desired specific location in vivo by application of an external magnetic field (12). In general, MNPs are used as drug carriers by binding antibodies (13) and chemotherapeutic drugs (14). Commonly, chemotherapeutic drugs are loaded in MNPs, and they are involved in cancer treatment. MNPs in the field of cancer therapy are generally used in several different ways: Chemotherapy; magnetic hyperthermia (MHT) (15); photodynamic therapy (PDT) (16); and photothermal therapy (PTT) (17). In order to achieve an improved therapeutic effect, the general method used is combination therapy. The present review provides a framework for the application of MNPs in medicine, such as cancer diagnosis, drug delivery and treatment. Furthermore, nanotoxicity is also reviewed, as well as the potential challenges and the opportunities.

\section{Cancer diagnosis}

When cancer is discovered earlier, the cure rate is greatly improved. Therefore, early detection and timely diagnosis of cancer is key to reduce the mortality rate of patients (18). Tumor imaging technology has an important role in cancer diagnosis and the choice of late clinical treatment options. Furthermore, MNPs are the contrast agents that are most widely researched and used in cancer imaging. Here, the imaging methods and the imaging positions of MNPs are discussed. 


\section{Imaging methods}

$M R I$. Due to the high spatial resolution and tomographic capabilities of MRI, it has been considered to be one of the most valuable noninvasive imaging techniques (19), and MNPs have been recently proposed as a contrast agent for MRI (20). In general, in order to overcome the colloidal instability of MNPs, it is necessary to conduct surface modification of nanoparticles (NPs) by inducing the magnetic dipole interaction and its intrinsic surface energy (21). For example, water-dispersible polyethyleneimine (PEI)-coated $\mathrm{Fe}_{3} \mathrm{O}_{4}$ NPs were prepared, and then sequentially modified with PEGylated folic acid (FA) and fluorescein isothiocyanate (FI) via PEI mediated conjugation chemistry (21). The remaining PEI surface amines were subjected to acetylation to form the colloidally stable FA-functionalized $\mathrm{Fe}_{3} \mathrm{O}_{4} \mathrm{NPs}$ for MRI (21). In MRI, research has demonstrated that early lesion detection of a 6-year old boy with glioblastoma multiforme may be achieved by sensitive imaging of superparamagnetic NPs or aggregates (22). Furthermore, research on Au nanorod@polypyrrole@iron oxide (Au NR@PPy@FexO) nanocomposites has demonstrated that these systems exhibit a low $\mathrm{r} 2 / \mathrm{r} 1$ ratio of 4.8 , making them efficient T1 positive contrast-enhancing agents for MRI (19). The study also indicated that the multifunctional nanocomposites exhibited the potential of the combination of therapeutic and diagnostic features (23).

Other imaging methods. A study by Mariappan et al (6) presented magneto acoustic tomography, which uses magnetomotive force due to a short pulsed magnetic field to induce ultrasound in SPION-labeled tissue and estimates an image of the distribution of the NPs in vivo with ultrasound imaging resolution. Magnetic particle imaging (MPI) allows high spatial resolution and sensitivity as a tomographic imaging technology. A study by Lindemann et al (24) suggested that University of Luebeck Dextran-coated SPIONs are a promising tracer material for use in innovative tumor cell analysis in MPI. In addition, photoacoustic imaging has gained increasing attention as a potential imaging tool. A study by Li et al (25) developed a new generation of magnetomotive photoacoustic featuring cyclic magnetic motion and ultrasound speckle tracking, whose imaging capture frame rate is several hundred times faster than the photoacoustic speckle tracking method that has previously been demonstrated (26). Stone et al (8) demonstrated a magnetic NP system that may be used to observe the NP fate within a biofilm using NIR imaging. Additionally, Xi et al (27) presented a breast imaging technique combining high-resolution NIR light induced photoacoustic tomography (PAT) with NIR dye-labeled amino-terminal fragments of urokinase plasminogen activator receptor-targeted magnetic iron oxide NPs (NIR830-ATF-IONP) for breast cancer imaging using a murine model of orthotopic mammary cancer. Other studies have demonstrated that ultrashort echo time (UTE) imaging (28) and MPI (29) may improve the detection of MNPs in cancer.

For MNPs, in addition to MRI, other imaging methods are used in combination. This dual imaging method may improve the accuracy of diagnosis. For instance, dual imaging of single-photon emission computed tomography (SPECT) and MRI has been utilized in pancreatic (4) and breast (30) cancer. A study by Jang et al (31) demonstrated the synthesis and the use of monodisperse iron oxide NPs coated with fluorescent silica nano-shells for fluorescence and magnetic resonance dual imaging of tumors. Furthermore, Sun et al (32) utilized MRI and optical imaging (OI) for the diagnosis of breast cancer.

\section{Imaging position}

Pancreas. Pancreatic cancer is one of the most lethal cancers in the world due to its late presentation (33). Therefore, early diagnosis will increase the cure rate of patients. When chitosan-coated MNPs and survivin antisense oligonucleotides (ASON) are conjugated to give Sur-MNPs, the MNPs functionalized with ASON lead to targeted localization in pancreatic tumors (34). Survivin-targeted NPs could be used by MRI for detection of pancreatic tumors (34). Taking into account the more sensitive diagnostic tools to allow early medical imaging, biodegradable NPs prepared using recombinant human serum albumin and incorporated iron oxide (maghemite, $\gamma$-Fe2O3) NPs were developed (4). Improved targeting and imaging properties were demonstrated in mice using SPECT-CT and MRI (4).

Breast. A study by Bucci et al (35) outlined some guidelines for the design of the imaging device for MNPs to enhance the microwave imaging of breast cancer. The results demonstrated that MNP-enhanced microwave imaging may reliably detect cancer lesions even using low-complexity arrangements, when designed according to the devised guidelines. An investigation by Kato et al (36) concluded that liposome encapsulation significantly improved the delivery and retention of SPIONs in breast tumors, and targeted SPION liposomes have significantly improved accumulation in breast tumors, which could be the optimal option for MRI detection of breast tumors. With magnetic nanoclusters coated with ruthenium (II) complexes doped with silica (fluorescent magnetic NPs; FMNPs), Sun et al (32) demonstrated that more peptide cyclic-arginine-glycine-aspartic acid (RGD)-FMNPs accumulated around the tumors than FMNPs. The result indicated the potential application of RGD-FMNPs as a targeting molecular probe for detection of breast cancer using MRI and OI (32). In order to make more accurate and specific diagnosis of breast cancer, Bevacqua and Scapaticci (37) adopted a compressive sensing approach for three-dimensional breast cancer microwave imaging. In their paper, an ad hoc compressive sensing algorithm was developed by exploiting the knowledge of the maximum concentration of MNPs that may be targeted in human tissues (37).

Prostate. MRI provides the best soft tissue resolution and has an important role in the management of prostate cancer as it is the recommended imaging modality for patients with prostate cancer (38). Sentinel lymph node (SLN) evaluation in patients with prostate cancer is commonly performed via lymphoscintigraphy following injection of radiolabeled tracers (39). For example, patients underwent MRI at $1.5 \mathrm{~T}$ before and 1 day after SPION injection using T1-, T2- and T2*-weighted sequences (39). This was the first study to use intraprostatic injection of SPIONs to visualize SLNs by MRI in patients with prostate cancer (39). A study by Winter et al (40) indicated that using a transrectal intraprostatic injection of SPIONs for magnetic marking in prostate cancer is safe, feasible and reliably identifies SLNs and lymph node metastases in the majority of patients. In other cases, diffusion-weighted (DW)-MRI is sensitive to water diffusion throughout tissues, which correlates with the Gleason score, a histological measure of prostate cancer aggressiveness (40). 
Table I. Specific circumstances of imaging and treatment modalities.

\begin{tabular}{|c|c|c|c|c|}
\hline Author, year & Imaging method & Treatment modality & Treatment site & (Refs.) \\
\hline Wang et al, 2014 & MRI & PDT & Head and neck & (44) \\
\hline Bhattacharya et al, 2016 & MRI & Targeted therapy & Head and neck & $(45)$ \\
\hline Pilapong et al, 2014 & MRI & Targeted therapy & Liver & $(46)$ \\
\hline Wu et al, 2015 & MRI & PTT & Liver/cervical & $(47)$ \\
\hline Azhdarzadeh et al, 2016 & MRI & PTT & Colon & $(48)$ \\
\hline Yu et al, 2015 & MRI/photoacoustic tomography & PTT & Liver/cervical & (49) \\
\hline Zhou et al, 2015 & MRI/photoacoustic imaging & Photothermal ablation & Breast & $(50)$ \\
\hline Lin et al, 2015 & MRI/fluorescence imaging & Targeted therapy & Cervical & $(51)$ \\
\hline Wang et al, 2014 & MRI/ fluorescent imaging & Targeted therapy & Stomach & (13) \\
\hline Li et al, 2015 & MRI/infrared thermal imaging & Chemotherapy/MHT & Liver & $(52)$ \\
\hline Kim et al, 2016 & MRI/optical imaging & PDT/MHT & Melanoma & (53) \\
\hline Sun et al, 2016 & MRI/computed tomography & Targeted therapy & Brain & $(54)$ \\
\hline Zhao et al, 2014 & Near-infrared fluorescence imaging & PDT & Head and neck & $(55)$ \\
\hline
\end{tabular}

MRI, magnetic resonance imaging; PDT, photodynamic therapy; PTT, photothermal therapy; MHT, magnetic hyperthermia.

The incorporation of DW-MRI-based prostate cancer stratification and monitoring could increase the accuracy of preclinical trials using mice with transgenic adenocarcinoma of the mouse prostate (41).

Lung. To improve the sensitivity of detection of metastasis of lung cancer, Wan et al (42) created immune SPIONs used in magnetic resonance immune imaging. These SPIONs were coated with oleic acid and carboxymethyl dextran, and then conjugated to mouse anti-cluster of differentiation (CD) 44v6 monoclonal antibody (42). The prepared SPIONs are potentially useful for lung tumor-targeting diagnosis. In addition, targeted pulmonary inhalation aerosol-based delivery facilitates the direct application of drugs to the lungs in a controlled manner, and has inherent advantages (43). Therefore, Nishimoto et al (29) investigated the feasibility of applying MPI to pulmonary imaging using nebulized MNPs and to quantify the mucociliary clearance in the lung, using small animal experiments.

Other. Common contrast agents are frequently used in the pancreas, breast and prostate. In other cases, the diagnosis and treatment are carried out in combination, with a variety of imaging modalities, as outlined in Table I.

\section{Drug delivery}

Magnetic targeted drug delivery has been used to improve the therapeutic performance of drugs and reduce the side effects associated with the conventional treatment of cancer. MNPs coated with a stabilizing shell have been successfully used as contrast agents for MRI (56). On the basis of early diagnosis, the treatment may be carried out at the same time, and the efficiency may be greatly improved. Therefore, MNPs are essential for drug delivery. By binding antibodies, chemotherapeutics or other drugs, MNPs may be used as drug carriers.
Antibodies. Research has demonstrated that antibody-conjugated MNPs may not only be used for detection of ovarian cancer biomarkers, but may also treat ovarian cancer due to their high-level accumulation within cancer cells $(57,58)$. A study by Wang et al (13) reported, for the first time, that anti- $\alpha$-subunit of adenosine triphosphate synthase antibody, HAI-178 monoclonal antibody (mAb)-conjugated fluorescent MNPs, was successfully used for targeted imaging and simultaneous therapy of in vivo gastric cancer. As for human breast cancer, Shanehsazzadeh et al (59) demonstrated disappointing in vivo results that had very low accumulation of nanoprobes in the targeted site when conjugating ultra-small SPIONs with C595 mAb. On the contrary, a study by Rasaneh and Dadras (60) suggested that combining MNPs and a permanent magnet may increase the therapeutic efficacy of herceptin for increased accumulation in the tumor site.

In order to improve the therapeutic efficiency, the combination of antibodies and chemotherapeutic drugs is attracting increasing attention. A study by Aires et al (61) presented a novel multi-functionalized iron oxide MNP with anti-CD44 antibody and gemcitabine derivatives, and their application for the selective treatment of CD44-positive cancer cells. In addition, Huang et al (62) developed an ovarian cancer dual-targeting therapy involving magnetic $\mathrm{Fe}_{3} \mathrm{O}_{4} \mathrm{NPs}$ grafted with single-chain antibody and docetaxel loaded $\beta$-cyclodextrin. These studies have demonstrated the great potential of the combination of antibodies and chemotherapeutic drugs.

Chemotherapeutic drugs. Commonly used chemotherapy drugs include doxorubicin (DOX), paclitaxel, cisplatin, gemcitabine, methotrexate, docetaxel, sorafenib and mitomycin $\mathrm{C}$, as outlined in Table II. DOX is the most widely applied chemotherapy drug in targeted delivery systems (63). MNPs have limits in their stability because of hydrophobic coating (64). To address this issue, a reducible copolymer self-assembled 
Table II. Various chemotherapeutic drugs delivered through magnetic nanoparticles.

\begin{tabular}{|c|c|c|c|c|c|}
\hline Author, year & Drug & Polymer modification & Average size, $\mathrm{nm}$ & Cancer cell line & (Refs.) \\
\hline Zohreh et al, 2016 & Doxorubicin & $\begin{array}{l}\text { Starch-g-poly (methyl } \\
\text { methacrylate-co-PEG- } \\
\text { acrylamide) }\end{array}$ & 93 & HeLa & (67) \\
\hline Frounchi and Shamshiri, 2015 & & Poly (lactic acid)/PEG & 22 & - & $(68)$ \\
\hline Bhattacharya et al, 2016 & & $\begin{array}{l}\text { Polyethyleneimine cross- } \\
\text { linked Pluronic F127 } \\
\text { copolymer }\end{array}$ & $\sim 91$ & HeLa & $(45)$ \\
\hline Pilapong et al, 2014 & & Carboxymethyl & 5 & K562/ADR & (69) \\
\hline Wu et al, 2014 & & APS-PEG-TFEE & 20 & MCF-7 & (70) \\
\hline Chandra et al, 2015 & & $\begin{array}{l}\text { L6-PEG-PAMAM and } \\
\text { S6-PEG-PAMAM }\end{array}$ & $9.6 \pm 0.13$ & - & (71) \\
\hline Hałupka-Bryl et al, 2015 & & $\begin{array}{l}\text { PEG-poly } \\
\text { (4-chloromethylstyrene) }\end{array}$ & $8-12$ & - & $(72)$ \\
\hline Zou et al, 2015 & & Chitosan & $\sim 120$ & $\mathrm{MCF}-7$ & (73) \\
\hline Tansik et al, 2014 & & PLGA & 74 & MCF-7 & (74) \\
\hline Ghorbani et al, 2016 & & $\begin{array}{l}\text { Poly } \\
\text { (N-isopropylacrylamide-co-IA) }\end{array}$ & 30 & HeLa & $(75)$ \\
\hline Mangaiyarkarasi et al, 2016 & Paclitaxel & Chitosan & $19-37$ & A549 & (76) \\
\hline Lin et al, 2015 & & $\begin{array}{l}\text { Poly[(N-isopropylacrylamide } \\
\text {-r-acrylamide)-b-L-lactic acid] }\end{array}$ & $229.0 \pm 13.2$ & HeLa & (77) \\
\hline Fazilati, 2014 & Cisplatin & Heparin & $45 \pm 15$ & CP70 & (78) \\
\hline Parsian et al, 2016 & Gemcitabine & Chitosan & 4 & SKBR; MCF-7 & (79) \\
\hline Roy et al, 2016 & Methotrexate & $\begin{array}{l}\text { Poly (N-isopropyl } \\
\text { acrylamide)-co-tyrosine }\end{array}$ & $10-15$ & MCF-7 & (80) \\
\hline Nagesh et al, 2016 & Docetaxel & $\begin{array}{l}\text { Cyclodextrin and } \\
\text { F127 polymer }\end{array}$ & $139.5 \pm 2.16$ & $\mathrm{C} 4-2$ & $(81)$ \\
\hline Li et al, 2015 & Sorafenib & PEGylated PLGA & $205 \pm 3.12$ & BEL7402 & (82) \\
\hline Türkmen et al, 2014 & Mitomycin C & $\begin{array}{l}\text { Poly (hydroxyethyl } \\
\text { methacrylate) }\end{array}$ & 200 & - & (83) \\
\hline Unsoy et al, 2014 & Bortezomib & Chitosan & $5-7$ & HeLa; SiHa & (84) \\
\hline
\end{tabular}

PEG, polyethylene glycol; APS-PEG-TFEE, Polyethylene glycol dicarboxylic acid; PLGA, Poly (DL-lactic-co-glycolic acid).

with SPIONs was developed to deliver DOX for cancer therapy (14). The copolymer of reducible polyamidoamine with polyethylene glycol/dodecyl amine graft was synthesized by Michael addition (14). Additionally, research has demonstrated that the application of iron oxide MNPs improved DOX-NP cell penetration compared to free DOX and achieved a cellular response to DOX-NP conjugates similar to that of DOX alone (65). SPIONs have also been investigated as a carrier for targeted drug delivery. For example, Cicha et al (66) set up an in vitro system to analyze the different aspects of cellular responses to mitoxantrone-carrying SPIONs and to the drug released from SPIONs.

Other. In addition to chemotherapy drugs, some traditional Chinese medicine monomers have been utilized as anti-cancer drugs in targeted drug delivery. A study by Nigam and Bahadur (85) demonstrated the fabrication and characterization of dendrimerized MNPs as delivery vectors for epigallocatechin gallate. Recently, curcumin has been widely applied in the drug delivery of MNPs in breast and ovarian cancer. A study by Mancarella et al (86) developed a layer by layer functionalization of $\mathrm{Fe}_{3} \mathrm{O}_{4}$ NPs by coating them in Dextran and Poly(L-lysine), which obtained a high upload of curcumin in $\mathrm{Fe}_{3} \mathrm{O}_{4}$ NPs for treating ovarian cancer. Furthermore, magnetic $\mathrm{Fe}_{3} \mathrm{O}_{4} @$ zirconium phosphate core-shell NPs and magnetic $\mathrm{Fe}_{3} \mathrm{O}_{4} @$ @ydroxyapatite-PEI-b-cyclodextrin NPs have been demonstrated to be effective drug carriers for the delivery of curcumin, and these were both used to treat breast cancer $(87,88)$.

In other cases, oligonucleotides are applied in drug delivery. A study by Pourianazar and Gunduz (89) utilized three-layer MNPs composed of a $\mathrm{Fe}_{3} \mathrm{O}_{4}$ magnetic core, an aminosilane interlayer and a cationic poly(amidoamine) dendrimer, which enhanced the accumulation of $\mathrm{CpG}$-oligodeoxynucleotides 
molecules in tumor cells as a novel targeted delivery system. Furthermore, 2-amino-2-deoxy-glucose was conjugated to -COOH-modified cobalt ferrite MNPs, which were designed to target tumor cells as a potential targetable drug/gene delivery agent for cancer treatment (90). In summary, MNPs may provide a high-efficiency drug delivery system with the potential to achieve drug targeting.

\section{Cancer treatment}

As a drug carrier, the ultimate goal of MNPs is to treat. This section discusses and provides examples of the use of MNPs in MHT, PDT, PTT and combined treatment.

MHT. MHT has attracted a lot of interest in recent years due to its potential use in medicine. MNPs are able to convert electromagnetic energy into heat (91). Therefore, the most popular application for MNPs is most likely the destruction of tumor cells by heating them to their apoptosis threshold (92). Magnetic field strength and frequency, NP size, NP concentration and solution viscosity are important parameters and may improve the efficiency of heat generation for effective cancer therapy while administering a low NP treatment dose (93). Although MNPs represent an area of active development for MHT, the in vivo anti-tumor effect under a low-frequency magnetic field using MNPs has not yet been demonstrated. A study by Cheng et al (94) demonstrated the successful use of spin-vortex, disk-shaped permalloy magnetic particles in a low-frequency, rotating magnetic field for the in vitro and in vivo destruction of glioma cells. In addition, the hysteresis loss is also important for MHT, as increasing the hysteresis loss is improving the heating efficiency (94). A study by Sasayama et al (95) examined the hysteresis loss of magnetically fractionated MNPs for hyperthermia application. They concluded that the efficiency of hyperthermia is improved by magnetically separating MNPs (95). Generally, MHT may enhance the efficacy of chemotherapeutic drugs to some degree. For instance, by combining SPIONs (MF66) functionalized with Nucant multivalent pseudopeptide (N6L), DOX and MHT, the therapeutic effects of MHT in breast cancer could be strongly enhanced (96). Furthermore, dual-functional Pt-Fe-hydroxyapatite MNPs were developed for chemo-hyperthermia treatment of lung cancer (97). In addition, research has indicated that MHT of MNPs enhanced radiation therapy in murine models of human prostate cancer (98). In other cases, gene delivery also has an important role in MHT $(99,100)$.

$P D T$. PDT is an externally-activated and minimally invasive modality for cancer treatment. The process of PDT involves the systemic or local application of photosensitizing drugs, called photosensitizers (PSs), followed by photoexcitation of the PSs in the tissue using light of the appropriate wavelength and power (16). In the presence of oxygen, the PS is excited from the ground state to the excited state following activation with light of an appropriate wavelength, and an electron is transferred to nearby tissue oxygen, producing oxygen free radicals or excited singlet oxygen (101). These substances are also known as reactive oxygen species (ROS) $(102,103)$, which cause cell damage, and eventually lead to cancer tissue damage. To enhance the effect of PSs, building a targeted drug delivery system with MNPs has become of interest. For instance, a study by Park et al (104) synthesized multifunctional cobalt ferrite $\left(\mathrm{CoFe}_{2} \mathrm{O}_{4}\right) \mathrm{NPs}\left[\mathrm{CoFe}_{2} \mathrm{O}_{4}\right.$-hematoporphyrins (HPs)-FAs] functionalized by coating them with HP for introducing photo-functionality and by conjugating with FA for targeting cancer cells. Furthermore, other research has revealed that the $\mathrm{Fe}_{3} \mathrm{O}_{4} @ \mathrm{HP}$ particles demonstrated remarkable and efficient photodynamic anticancer activity, and exhibited strong anticancer effects on human prostate cancer (PC-3) and breast cancer (MDA-MB-231) cell lines (105). Pyropheophorbide-a (PPA) as a novel chlorin PS was prepared for PDT. PPA-coated multifunctional magneto-fluorescent NPs, $\mathrm{Fe}_{3} \mathrm{O}_{4} @ \mathrm{SiO}_{2} @$ CS@PPA (MFCSPPA) were designed (106). The experiments demonstrated that MFCSPPA had strong photodynamic therapy activity and low dark toxicity, and cell viability of human HeLa cervical cancer cells was reduced to $18 \%$ following treatment with PDT (106).

PTT. As a light absorbent of low toxicity on skin and deep tissue penetration, NIR may directly kill cancer cells by PTT, which has become a controlled treatment method (107). PTT using photothermal agents in combination with NIR has also gained increasing attention for cancer treatment (108). An example of this is engineering phosphopeptide-decorated MNPs as efficient photothermal agents for solid tumor therapy (109). Notably, the photothermal effect of MNP clusters was initially reported for the photothermal ablation (PTA) of tumors in vitro and in vivo. Compared with individual magnetic $\mathrm{Fe}_{3} \mathrm{O}_{4} \mathrm{NPs}$, clustered $\mathrm{Fe}_{3} \mathrm{O}_{4}$ NPs may result in a marked increase in NIR absorption (17). Upon NIR irradiation at $808 \mathrm{~nm}$, clustered $\mathrm{Fe}_{3} \mathrm{O}_{4}$ NPs inducing higher temperatures were more cytotoxic against A549 cells (17). In the majority of cases, PTT and MRI are carried out in combination $(110,111)$. However, a study indicated that, compared with their large counterparts, small $\mathrm{Fe}_{3} \mathrm{O}_{4}$ NPs exhibited greater cellular internalization, thus enabling a higher PTA efficacy in vitro (112). In addition, $120 \mathrm{~nm}$ may be the optimal diameter of $\mathrm{Fe}_{3} \mathrm{O}_{4}$ NPs for MRI and PAT in vitro (112). Therefore, the size of MNPs may be an important factor for PTT.

Combined treatment. In general, MNPs act as drug carriers for targeted delivery systems. The nanocomplex with PTT agents and PSs together may be used for combined cancer PTT and PDT. A study by Bhana et al (113) demonstrated the first application of magnetic-optical hybrid nanosystems for magnetic-field-guided drug delivery and dual mode PTT and PDT. The composite NPs may generate heat and ROS simultaneously upon NIR laser irradiation, and may even be selectively delivered to the mitochondria (114). Furthermore, MNPs have been demonstrated to have the dual capacity to act as both magnetic and PTT agents for amplification of heating efficiency (115). In conclusion, these results demonstrated high accumulation of MNPs in tumors and excellent tumor regression.

\section{Conclusions and perspectives}

MNPs as contrast agents of imaging have a great potential in adjuvant therapy. However, due to some toxicity being associated with the use of MNPs, many restrictions have been 
applied in their application. Surface coatings of MNPs are known to influence advantageous features of MNPs, as well as potential toxicity. Research has indicated that the larger the size of the MNPs, the greater the accumulation in vivo. Therefore, controlling the size and surface coatings of MNPs could reduce toxicity and improve magnetic behaviors.

In the present review, we focused on cancer diagnosis by imaging, drug delivery and treatment using MNPs. Despite many successful studies using MNPs as a theranostic material, there are still some challenges. While many MNP formulations have demonstrated excellent results in small animal models, they cannot reach the clinical requirement. By focusing on improving their drug loading capacity, and increasing their specificity and affinity to target cancer cells, MNPs may become suitable for clinical use with integrated imaging and multimodal therapy in the near future and dramatically impact the treatment of cancer.

\section{Acknowledgements}

The present review was supported by the National Natural Science Foundation of China (grant no. 81473361).

\section{References}

1. Yarar E, Karakas G, Rende D, Ozisik R and Malta S: Influence of surface coating of magnetic nanoparticles on mechanical properties of polymer nanocomposites. Journal, 2016.

2. Briguet A, Jeandey $C$ and Tournier E: System of coils for producing additional fields for obtaining polarization fields with constant gradients in a magnet having polarization pole pieces for image production by nuclear magnetic resonance. Journal, 1988.

3. Dan M, Bae Y, Pittman TA and Yokel RA: Alternating magnetic field-induced hyperthermia increases iron oxide nanoparticle cell association/uptake and flux in blood-brain barrier models. Pharm Res 32: 1615-1625, 2015.

4. Rosenberger I, Strauss A, Dobiasch S, Weis C, Szanyi S, Gil-Iceta L, Alonso E, González Esparza M, Gómez-Vallejo V, Szczupak B, et al: Targeted diagnostic magnetic nanoparticles for medical imaging of pancreatic cancer. J Control Release 214: 76-84, 2015.

5. Lu AH, Zhang XQ, Sun Q, Zhang Y, Song Q, Schüth F, Chen C and Cheng F: Precise synthesis of discrete and dispersible carbon-protected magnetic nanoparticles for efficient magnetic resonance imaging and photothermal therapy. Nano Res 9: 1460-1469, 2016.

6. Mariappan L, Shao Q, Jiang C, Yu K, Ashkenazi S, Bischof JC and He B: Magneto acoustic tomography with short pulsed magnetic field for in-vivo imaging of magnetic iron oxide nanoparticles. Nanomedicine 12: 689-699, 2016.

7. FitzGerald PF, Butts MD, Roberts JC, Colborn RE, Torres AS, Lee BD, Yeh BM and Bonitatibus PJ Jr: A proposed computed tomography contrast agent using carboxybetaine zwitterionic tantalum oxide nanoparticles: Imaging, biological and physicochemical performance. Invest Radiol 51: 786-796, 2016.

8. Stone RC, Fellows BD, Qi B, Trebatoski D, Jenkins B, Raval Y, Tzeng TR, Bruce TF, McNealy T, Austin MJ, et al: Highly stable multi-anchored magnetic nanoparticles for optical imaging within biofilms. J Colloid Interface Sci 459: 175-182, 2015.

9. Zhou Q and Wei Y: For better or worse, iron overload by superparamagnetic iron oxide nanoparticles as a MRI contrast agent for chronic liver diseases. Chem Res Toxicol 30: 73-80, 2017.

10. Tse BW, Cowin GJ, Soekmadji C, Jovanovic L, Vasireddy RS, Ling MT, Khatri A, Liu T, Thierry B and Russell PJ: PSMA-targeting iron oxide magnetic nanoparticles enhance MRI of preclinical prostate cancer. Nanomedicine (Lond) 10 375-386, 2015.

11. Ahmed M and Douek M: The role of magnetic nanoparticles in the localization and treatment of breast cancer. Biomed Res Int 2013: 281230, 2013.
12. Singh A, Dilnawaz F, Mewar S, Sharma U, Jagannathan N and Sahoo SK: Retraction notice for composite polymeric magnetic nanoparticles for codelivery of hydrophobic and hydrophilic anticancer drugs and MRI imaging for cancer therapy. ACS Appl Mater Interf 6: 4595, 2014

13. Wang C, Bao C, Liang S, Zhang L, Fu H, Wang Y, Wang K, Li C, Deng M, Liao Q, et al: HAI-178 antibody-conjugated fluorescent magnetic nanoparticles for targeted imaging and simultaneous therapy of gastric cancer. Nanoscale Res Lett 9: 274,2014

14. Chen J, Shi M, Liu P, Ko A, Zhong W, Liao W and Xing MM: Reducible polyamidoamine-magnetic iron oxide self-assembled nanoparticles for doxorubicin delivery. Biomaterials 35 : 1240-1248, 2014.

15. Yin PT, Shah BP and Lee KB: Combined magnetic nanoparticle-based microRNA and hyperthermia therapy to enhance apoptosis in brain cancer cells. Small 10: 4106-4112, 2014.

16. Li L, Nurunnabi M, Nafiujjaman M, Jeong YY, Lee Yk and Huh KM: A photosensitizer-conjugated magnetic iron oxide/gold hybrid nanoparticle as an activatable platform for photodynamic cancer therapy. J Mat Chem B 2: 2929-2937, 2014.

17. Shen S, Wang S, Zheng R, Zhu X, Jiang X, Fu D and Yang W: Magnetic nanoparticle clusters for photothermal therapy with near-infrared irradiation. Biomaterials 39: 67-74, 2015.

18. Zhang H, Li J, Hu Y, Shen M, Shi X and Zhang G: Folic acid-targeted iron oxide nanoparticles as contrast agents for magnetic resonance imaging of human ovarian cancer. J Ovarian Res 9: 19, 2016.

19. Luo Y, Yang J, Yan Y, Li J, Shen M, Zhang G, Mignani S and Shi X: RGD-functionalized ultrasmall iron oxide nanoparticles for targeted $\mathrm{T}_{1}$-weighted MR imaging of gliomas. Nanoscale 7: 14538-14546, 2015.

20. Yadollahpour A, Hosseini SA, Rashidi S and Farhadi F: Applications of magnetic nanoparticles as contrast agents in MRI: Recent advances and clinical challenges. Int J Pharm Res Allied Sci, 2016.

21. Li J, Hu Y, Yang J, Sun W, Cai H, Wei P, Sun Y, Zhang G, Shi X and Shen M: Facile synthesis of folic acid-functionalized iron oxide nanoparticles with ultrahigh relaxivity for targeted tumor MR imaging. J Mater Chem B 3: 5720-5730, 2015.

22. Li Z, Hsu CH, Dimitrov N, Hwang DW, Chang HW, Hwang LP and Lin YY: Sensitive imaging of magnetic nanoparticles for cancer detection by active feedback MR. Magn Reson Med: Apr 4, 2015 (Epub ahead of print).

23. Yang Z, Ding $X$ and Jiang $J$ : Facile synthesis of magnetic-plasmonic nanocomposites as $\mathrm{T}_{1}$ MRI contrast enhancing and photothermal therapeutic agents. Nano Res 9: 787-799, 2016.

24. Lindemann A, Lüdtke-Buzug K, Fräderich BM, Gräfe K, Pries R and Wollenberg B: Biological impact of superparamagnetic iron oxide nanoparticles for magnetic particle imaging of head and neck cancer cells. Int J Nanomedicine 9: 5025-5040, 2014.

25. Li J, Arnal B, Wei CW, Shang J, Nguyen TM, O'Donnell M and Gao X: Magneto-optical nanoparticles for cyclic magnetomotive photoacoustic imaging. ACS Nano 9: 1964-1976, 2015.

26. Jaeger M, Preisser S, Kitz M, Ferrara D, Senegas S, Schweizer D and Frenz M: Improved contrast deep optoacoustic imaging using displacement-compensated averaging: Breast tumour phantom studies. Phys Med Biol 56: 5889-5901, 2011.

27. Xi L, Grobmyer SR, Zhou G, Qian W, Yang L and Jiang H: Molecular photoacoustic tomography of breast cancer using receptor targeted magnetic iron oxide nanoparticles as contrast agents. J Biophotonics 7: 401-409, 2014.

28. Wang L, Zhong X, Qian W, Huang J, Cao Z, Yu Q, Lipowska M, Lin R, Wang A, Yang L and Mao H: Ultrashort echo time (UTE) imaging of receptor targeted magnetic iron oxide nanoparticles in mouse tumor models. J Magn Reson Imaging 40: 1071-1081, 2014.

29. Nishimoto K, Mimura A, Aoki M, Banura N and Murase K: Application of magnetic particle imaging to pulmonary imaging using nebulized magnetic nanoparticles. Open J Med Imag 5: 49, 2015

30. Deng S, Zhang W, Zhang B, Hong R, Chen Q, Dong J, Chen Y, Chen Z and Wu Y: Radiolabeled cyclic arginine-glycine-aspartic (RGD)-conjugated iron oxide nanoparticles as single-photon emission computed tomography (SPECT) and magnetic resonance imaging (MRI) dual-modality agents for imaging of breast cancer. J Nano Res 17: 19, 2015. 
31. Jang H, Lee C, Nam GE, Quan B, Choi HJ, Yoo JS and Piao Y: In vivo magnetic resonance and fluorescence dual imaging of tumor sites by using dye-doped silica-coated iron oxide nanoparticles. J Nano Res 18: 1-11, 2016.

32. Sun J, Teng ZG, Tian Y, Wang JD, Guo Y, Kim DH, Larson AC and $\mathrm{Lu}$ GM: Targeted fluorescent magnetic nanoparticles for imaging of human breast cancer. Int J Clin Exp Med 7: 4747-4758, 2014

33. Karelia D, Pandey MK, Irby R, Amin S and Sharma AK Abstract 3439: Suppression of pancreatic cancer cell growth by NISC- 6 through activation of Par-4 and death receptor 5. Exp Mol Ther 73, 2013

34. Wang Z, Tong M, Chen X, Hu S, Yang Z, Zhang Y, Zhou H, Wu Y, Li X and Li D: Survivin-targeted nanoparticles for pancreatic tumor imaging in mouse model. Nanomedicine 12: 1651-1661, 2016.

35. Bucci OM, Crocco L and Scapaticci R: On the optimal measurement configuration for magnetic nanoparticles-enhanced breast cancer microwave imaging. IEEE Trans Biomed Eng 62: 407-414, 2015.

36. Kato Y, Zhu W, Backer MV, Neoh CC, Hapuarachchige S, Sarkar SK, Backer JM and Artemov D: Noninvasive imaging of liposomal delivery of superparamagnetic iron oxide nanoparticles to orthotopic human breast tumor in mice. Pharm Res 32: 3746-3755, 2015 .

37. Bevacqua MT and Scapaticci R: A Compressive sensing approach for $3 \mathrm{D}$ breast cancer microwave imaging with magnetic nanoparticles as contrast agent. IEEE Trans Med Imaging 35: 665-673, 2016.

38. Guneyli S, Erdem CZ and Erdem LO: Magnetic resonance imaging of prostate cancer. Clin Imaging 40: 601-609, 2016.

39. Winter A, Kowald T, Paulo T, Goos P, Engels S, Gerullis H Chavan A and Wawroschek F: 1060 Magnetic resonance sentinel lymph node imaging in prostate cancer using intraprostatic injection of superparamagnetic iron oxide nanoparticles: The first in-human results. Europ Urol Suppl 15: e1060, 2016.

40. Winter A, Woenkhaus J and Wawroschek F: 979 Magnetic marking and intraoperative detection of primary draining lymph nodes in prostate cancer using intraprostatic injection of superparamagnetic iron oxid nanoparticles. Europ Urol Suppl 14: e979, 2015.

41. Hill DK, Kim E, Teruel JR, Jamin Y, Widerøe M, Søgaard CD Størkersen $\varnothing$, Rodrigues DN, Heindl A, Yuan Y, et al: Diffusion-weighted MRI for early detection and characterization of prostate cancer in the transgenic adenocarcinoma of the mouse prostate model. J Magn Reson Imaging 43: 1207-1217, 2016.

42. Wan X, Song Y, Song N, Li J, Yang L, Li Y and Tan H: The preliminary study of immune superparamagnetic iron oxide nanoparticles for the detection of lung cancer in magnetic resonance imaging. Carbohydr Res 419: 33-40, 2016.

43. Stocke NA, Meenach SA, Arnold SM, Mansour HM and Hilt JZ: Formulation and characterization of inhalable magnetic nanocomposite microparticles (MnMs) for targeted pulmonary delivery via spray drying. Int J Pharm 479: 320-328, 2015

44. Wang D, Fei B, Halig LV, Qin X, Hu Z, Xu H, Wang YA Chen Z, Kim S, Shin DM and Chen ZG: Targeted iron-oxide nanoparticle for photodynamic therapy and imaging of head and neck cancer. ACS Nano 8: 6620-6632, 2014.

45. Bhattacharya D, Behera B, Sahu SK, Ananthakrishnan R, Maiti TK and Pramanik P: Design of dual stimuli responsive polymer modified magnetic nanoparticles for targeted anti-cancer drug delivery and enhanced MR imaging. New J Chem 40: 545-557, 2016

46. Pilapong C, Sitthichai S, Thongtem S and Thongtem T: Smart magnetic nanoparticle-aptamer probe for targeted imaging and treatment of hepatocellular carcinoma. Int J Pharm 473 469-474, 2014

47. Wu M, Zhang D, Zeng Y, Wu L, Liu X and Liu J: Nanocluster of superparamagnetic iron oxide nanoparticles coated with poly (dopamine) for magnetic field-targeting, highly sensitive MRI and photothermal cancer therapy. Nanotechnology 26: 115102, 2015.

48. Azhdarzadeh M, Atyabi F, Saei AA, Varnamkhasti BS, Omidi Y, Fateh M, Ghavami M, Shanehsazzadeh S and Dinarvand R: Theranostic MUC-1 aptamer targeted gold coated superparamagnetic iron oxide nanoparticles for magnetic resonance imaging and photothermal therapy of colon cancer. Colloids Surf B Biointerfaces 143: 224-232, 2016.
49. Yu J, Yin W, Zheng X, Tian G, Zhang X, Bao T, Dong X, Wang Z $\mathrm{Gu} Z$, Ma X and Zhao Y: Smart MoS2/Fe3O4 nanotheranostic for magnetically targeted photothermal therapy guided by magnetic resonance/photoacoustic imaging. Theranostics 5: 931-935, 2015

50. Zhou M, Singhana B, Liu Y, Huang Q, Mitcham T, Wallace MJ, Stafford RJ, Bouchard RR and Melancon MP: Photoacousticand magnetic resonance-guided photothermal therapy and tumor vasculature visualization using theranostic magnetic gold nanoshells. J Biomed Nanotechnol 11: 1442-1450, 2015.

51. Lin J, Li Y, Li Y, Wu H, Yu F, Zhou S, Xie L, Luo F, Lin C and Hou Z: Drug/dye-loaded, multifunctional PEG-chitosan-iron oxide nanocomposites for methotraxate synergistically self-targeted cancer therapy and dual model imaging. ACS Appl Mater Interfaces 7: 11908-11920, 2015.

52. Li J, Hu Y, Hou Y, Shen X, Xu G, Dai L, Zhou J, Liu Y and Cai K: Phase-change material filled hollow magnetic nanoparticles for cancer therapy and dual modal bioimaging. Nanoscale 7 : 9004-9012, 2015

53. Kim KS, Kim J, Lee JY, Matsuda S, Hideshima S, Mori Y, Osaka $\mathrm{T}$ and $\mathrm{Na} \mathrm{K}$ : Correction: Stimuli-responsive magnetic nanoparticles for tumor-targeted bimodal imaging and photodynamic/hyperthermia combination therapy. Nanoscale 8: 12843,2016

54. Sun L, Joh DY, Al-Zaki A, Stangl M, Murty S, Davis JJ, Baumann BC, Alonso-Basanta M, Kaol GD, Tsourkas A and Dorsey JF: Theranostic application of mixed gold and superparamagnetic iron oxide nanoparticle micelles in glioblastoma multiforme. J Biomed Nanotechnol 12: 347-356, 2016.

55. Zhao X, Chen Z, Zhao H, Zhang D, Tao L and Lan M: Multifunctional magnetic nanoparticles for simultaneous cancer near-infrared imaging and targeting photodynamic therapy. RSC Adv 4: 62153-62159, 2014.

56. Khalkhali M, Rostamizadeh K, Sadighian S, Khoeini F, Naghibi $\mathrm{M}$ and Hamidi M: The impact of polymer coatings on magnetite nanoparticles performance as MRI contrast agents: A comparative study. Daru 23: 45, 2015.

57. Pal MK, Rashid M and Bisht M: Multiplexed magnetic nanoparticle-antibody conjugates (MNPs-ABS) based prognostic detection of ovarian cancer biomarkers, CA-125, $\beta-2 \mathrm{M}$ and ApoA1 using fluorescence spectroscopy with comparison of surface plasmon resonance (SPR) analysis. Biosens Bioelectron 73: 146-152, 2015.

58. Ndong C, Toraya-Brown S, Kekalo K, Baker I, Gerngross TU, Fiering SN and Griswold KE: Antibody-mediated targeting of iron oxide nanoparticles to the folate receptor alpha increases tumor cell association in vitro and in vivo. Int J Nanomedicine 10: 2595-2617, 2015.

59. Shanehsazzadeh S, Gruettner C, Lahooti A, Mahmoudi M, Allen BJ, Ghavami M, Daha FJ and Oghabian MA: Monoclonal antibody conjugated magnetic nanoparticles could target MUC-1-positive cells in vitro but not in vivo. Contrast Media Mol Imag 10: 225-236, 2014.

60. Rasaneh S and Dadras MR: The possibility of using magnetic nanoparticles to increase the therapeutic efficiency of Herceptin antibody. Biomed Tech (Berl) 60: 485-490, 2015.

61. Aires A, Ocampo SM, Simões BM, Josefa Rodríguez M, Cadenas JF, Couleaud P, Spence K, Latorre A, Miranda R, Somoza Á, et al: Multifunctionalized iron oxide nanoparticles for selective drug delivery to CD44-positive cancer cells Nanotechnology 27: 065103, 2016.

62. Huang X, Yi C, Fan Y, Zhang Y, Zhao L, Liang Z and Pan J: Magnetic $\mathrm{Fe}_{3} \mathrm{O}_{4}$ nanoparticles grafted with single-chain antibody ( $\mathrm{scFv}$ ) and docetaxel loaded $\beta$-cyclodextrin potential for ovarian cancer dual-targeting therapy. Mat Sci Eng. C 42 : 325-332, 2014

63. Jia Y, Yuan M, Yuan H, Huang X, Sui X, Cui X, Tang F, Peng J, Chen J, Lu S, et al: Co-encapsulation of magnetic Fe3O4 nanoparticles and doxorubicin into biodegradable PLGA nanocarriers for intratumoral drug delivery. Int J Nanomedicine 7: 1697-1708, 2012.

64. Singh M, Ulbrich P, Prokopec V, Svoboda P, Šantavá E and Štěpánek F: Effect of hydrophobic coating on the magnetic anisotropy and radiofrequency heating of $\gamma$-Fe2O3 nanoparticles. J Magnet Magn Mat 339: 106-113, 2013.

65. Augustin E, Czubek B, Nowicka AM, Kowalczyk A, Stojek Z and Mazerska Z: Improved cytotoxicity and preserved level of cell death induced in colon cancer cells by doxorubicin after its conjugation with iron-oxide magnetic nanoparticles. Toxicol In Vitro 33: 45-53, 2016 
66. Cicha I, Scheffler L, Ebenau A, Lyer S, Alexiou C and Goppelt-Struebe M: Mitoxantrone-loaded superparamagnetic iron oxide nanoparticles as drug carriers for cancer therapy: Uptake and toxicity in primary human tubular epithelial cells. Nanotoxicology 10: 557-566, 2016.

67. Zohreh N, Hosseini SH and Pourjavadi A: Hydrazine-modified starch coated magnetic nanoparticles as an effective $\mathrm{pH}$-responsive nanocarrier for doxorubicin delivery. J Indus Eng Chem 39: 203-209, 2016.

68. Frounchi M and Shamshiri S: Magnetic nanoparticles-loaded PLA/PEG microspheres as drug carriers. J Biomed Mater Res A 103: 1893-1898, 2015.

69. Pilapong C, Keereeta Y, Munkhetkorn S, Thongtem S and Thongtem T: Enhanced doxorubicin delivery and cytotoxicity in multidrug resistant cancer cells using multifunctional magnetic nanoparticles. Colloids Surf B: Bio 113: 249-253, 2014.

70. Wu J, Wang Y, Jiang W, Xu S and Tian R: Synthesis and characterization of recyclable clusters of magnetic nanoparticles as doxorubicin carriers for cancer therapy. Appl Surf Sci 321: $43-49,2014$

71. Chandra S, Noronha G, Dietrich S, Lang H and Bahadur D Dendrimer-magnetic nanoparticles as multiple stimuli responsive and enzymatic drug delivery vehicle. J Magn Magn Mat 380: 7-12, 2015

72. Hałupka-Bryl M, Bednarowicz M, Dobosz B, Krzyminiewski R, ZomaszT,WereszczyńskaB,NowaczykG,JarekMand NagasakiY: Doxorubicin loaded PEG-b-poly(4-vinylbenzylphosphonate) coated magnetic iron oxide nanoparticles for targeted drug delivery. J Magn Magn Mat 384: 320-327, 2015.

73. Zou Y, Liu P, Liu CH and Zhi XT: Doxorubicin-loaded mesoporous magnetic nanoparticles to induce apoptosis in breast cancer cells. Biomed Pharm 69: 355-360, 2015.

74. Tansık G, Yakar A and Gündüz U: Tailoring magnetic PLGA nanoparticles suitable for doxorubicin delivery. J Nano Res 16 : 2171, 2014.

75. Ghorbani M, Hamishehkar H, Arsalani N and Entezami AA Surface decoration of magnetic nanoparticles with folate-conjugated poly (N-isopropylacrylamide-co-itaconic acid): A facial synthesis of dual-responsive nanocarrier for targeted delivery of doxorubicin. Int J Poly Mat Poly Bio 65: 683-694, 2016

76. Mangaiyarkarasi R, Chinnathambi S, Karthikeyan S, Aruna P and Ganesan S: Paclitaxel conjugated Fe3O4@LaF3:Ce3+,Tb3+ nanoparticles as bifunctional targeting carriers for Cancer theranostics application. J Magn Magn Mat 399: 207-215, 2016.

77. Lin MM, Kang YJ, Sohn Y and Kim DK: Dual targeting strategy of magnetic nanoparticle-loaded and RGD peptide-activated stimuli-sensitive polymeric micelles for delivery of paclitaxel J Nano Res 17: 248, 2015.

78. Fazilati M: Anti-neoplastic applications of heparin coated magnetic nanoparticles against human ovarian cancer. J Inorg Organ Poly Mat 24: 551-559, 2014.

79. Parsian M, Unsoy G, Mutlu P, Yalcin S, Tezcaner A and Gunduz U: Loading of gemcitabine on chitosan magnetic nanoparticles increases the anti-cancer efficacy of the drug. Eur J Pharmacol 784: 121-128, 2016.

80. Roy E, Patra S, Madhuri R and Sharma PK: Stimuli-responsive poly (N-isopropyl acrylamide)-co-tyrosine@ gadolinium: Iron oxide nanoparticle-based nanotheranostic for cancer diagnosis and treatment. Coll Surf B: Bio 142: 248-258, 2016.

81. Nagesh PK, Johnson NR, Boya VK, Chowdhury P, Othman SF, Khalilzad-Sharghi V, Hafeez BB, Ganju A, Khan S, Behrman SW, et al: PSMA targeted docetaxel-loaded superparamagnetic iron oxide nanoparticles for prostate cancer. Colloids Surf B Biointerfaces 144: 8-20, 2016

82. Li YJ, Dong M, Kong FM and Zhou JP: Folate-decorated anticancer drug and magnetic nanoparticles encapsulated polymeric carrier for liver cancer therapeutics. Int J Pharm 489 83-90, 2015

83. Türkmen D, Bereli N, Corman ME, Shaikh H, Akgöl S and Denizli A: Molecular imprinted magnetic nanoparticles for controlled delivery of mitomycin C. Artif Cells Nanomed Biotechnol 42: 316-322, 2014

84. Unsoy G, Yalcin S, Khodadust R, Mutlu P, Onguru O and Gunduz U: Chitosan magnetic nanoparticles for $\mathrm{pH}$ responsive Bortezomib release in cancer therapy. Biomed Pharmacother 68: 641-648, 2014.

85. Nigam S and Bahadur D: Dendrimerized magnetic nanoparticles as carriers for the anticancer compound, epigallocatechin gallate. IEEE Transact on Magn 52: 1-5, 2016.
86. Mancarella S, Greco V, Baldassarre F, Vergara D, Maffia M and Leporatti S: Polymer-coated magnetic nanoparticles for curcumin delivery to cancer cells. Macromol Biosci 15: 1365-1374, 2015.

87. Kalita H, Rajput S, Kumar BP, Mandal M and Pathak A: Fe3O4@ zirconium phosphate core-shell nanoparticles for $\mathrm{pH}$-sensitive and magnetically guided drug delivery applications. RSC Adv 6: 21285-21292, 2016.

88. Akrami M, Khoobi M, Khalilvand-Sedagheh M, Haririan I, Bahador A, Faramarzi MA, Rezaei S, Javar HA, Salehi F, Ardestani SK and Shafiee A: Evaluation of multilayer coated magnetic nanoparticles as biocompatible curcumin delivery platforms for breast cancer treatment. RSC Adv 5: 88096-88107, 2015.

89. Pourianazar NT and Gunduz U: CpG oligodeoxynucleotide-loaded PAMAM dendrimer-coated magnetic nanoparticles promote apoptosis in breast cancer cells. Biomed Pharm 78: 81-91, 2016

90. Aşık E, Aslan TN, Volkan M and Güray NT: 2-Amino-2-deoxy-glucose conjugated cobalt ferrite magnetic nanoparticle (2DG-MNP) as a targeting agent for breast cancer cells. Environ Toxicol Pharmacol 41: 272-278, 2016.

91. Lee JH, Jang JT, Choi JS, Moon SH, Noh SH, Kim JW, Kim JG, Kim IS, Park KI and Cheon J: Exchange-coupled magnetic nanoparticles for efficient heat induction. Nat Nanotechnol 6: 418-422, 2011.

92. Guibert C, Dupuis V, Peyre V and Fresnais J: Hyperthermia of magnetic nanoparticles: Experimental study of the role of aggregation. J Phys Chem C 119: 28148-28154, 2015.

93. Shah RR, Davis TP, Glover AL, Nikles DE and Brazel CS: Impact of magnetic field parameters and iron oxide nanoparticle properties on heat generation for use in magnetic hyperthermia. J Magn Magn Mater 387: 96-106, 2015.

94. Cheng Y, Muroski ME, Petit DCMC, Mansell R, Vemulkar T, Morshed RA, Han Y, Balyasnikova IV, Horbinski CM, Huang $\mathrm{X}$, et al: Rotating magnetic field induced oscillation of magnetic particles for in vivo mechanical destruction of malignant glioma. J Control Release 223: 75-84, 2016.

95. Sasayama T, Yoshida T, Tanabe K, Tsujimura N and Enpuku K: Hysteresis loss of fractionated magnetic nanoparticles for hyperthermia application. IEEE Trans Magn 51: 1-4, 2015.

96. Kossatz S, Grandke J, Couleaud P, Latorre A, Aires A, Crosbie-Staunton K, Ludwig R, Dähring $\mathrm{H}$, Ettelt $\mathrm{V}$, Lazaro-Carrillo A, et al: Efficient treatment of breast cancer xenografts with multifunctionalized iron oxide nanoparticles combining magnetic hyperthermia and anti-cancer drug delivery. Breast Cancer Res 17: 66, 2015.

97. Tseng CL, Chang KC, Yeh MC, Yang KC, Tang TP and Lin FH: Development of a dual-functional Pt-Fe-HAP magnetic nanoparticles application for chemo-hyperthermia treatment of cancer. Ceram Int 40: 5117-5127, 2014.

98. Attaluri A, Kandala SK, Wabler M, Zhou H, Cornejo C, Armour M, Hedayati M, Zhang Y, DeWeese TL, Herman C and Ivkov R: Magnetic nanoparticle hyperthermia enhances radiation therapy: A study in mouse models of human prostate cancer. Int J Hyperthermia 31: 359-374, 2015.

99. Yin PT, Shah S, Pasquale NJ, Garbuzenko OB, Minko T and Lee KB: Stem cell-based gene therapy activated using magnetic hyperthermia to enhance the treatment of cancer. Biomaterials 81: 46-57, 2016

100. Yuan C, An Y, Zhang J, Li H, Zhang H, Wang L and Zhang D: Magnetic nanoparticles for targeted therapeutic gene delivery and magnetic-inducing heating on hepatoma. Nanotechnology 25: 345101, 2014.

101. Cheng J, Tan G, Li W, Li J, Wang Z and Jin Y: Preparation, characterization and in vitro photodynamic therapy of a pyropheophorbide-a-conjugated $\mathrm{Fe}_{3} \mathrm{O}_{4}$ multifunctional magnetofluorescence photosensitizer. RSC Adv 6: 37610-37620, 2016.

102. Hou W, Xia F, Alves CS, Qian X, Yang Y and Cui D: MMP2-targeting and redox-responsive PEGylated chlorin e6 nanoparticles for cancer near-infrared imaging and photodynamic therapy. ACS Appl Mat Interfaces 8: 1447-1457, 2016.

103. Li H, Song S, Wang $\mathrm{W}$ and Chen $\mathrm{K}$ : In vitro photodynamic therapy based on magnetic-luminescent Gd2O3:Yb,Er nanoparticles with bright three-photon up-conversion fluorescence under near-infrared light. Dalton Trans 44: 16081-16090, 2015.

104. Park BJ, Choi KH, Nam KC, Ali A, Min JE, Son H, Uhm HS, Kim HJ, Jung JS and Choi EH: Photodynamic anticancer activities of multifunctional cobalt ferrite nanoparticles in various cancer cells. J Biomed Nanotechnol 11: 226-235, 2015. 
105. Nam KC,Choi KH,Lee KD, Kim JH, Jung JS and Park BJ: Particle size dependent photodynamic anticancer activity of hematoporphyrin-conjugated $\mathrm{Fe}_{3} \mathrm{O}_{4}$ particles. J Nanomaterial 2016: 1, 2016.

106. Cheng J, Tan G, Li W, Zhang H, Wu X, Wang Z and Jin Y: Facile synthesis of chitosan assisted multifunctional magnetic Fe3O4@ SiO2@CS@pyropheophorbide-a fluorescent nanoparticles for photodynamic therapy. New J Chem 40: 8522-8534, 2016.

107. Cheng L, Yang K, Chen Q and Liu Z: Organic stealth nanoparticles for highly effective in vivo near-infrared photothermal therapy of cancer. ACS Nano 6: 5605-5613, 2012.

108.Liang X, Li Y, Li X, Jing L, Deng Z, Yue X, Li C and Dai Z: PEGylated polypyrrole nanoparticles conjugating gadolinium chelates for dual-modal MRI/Photoacoustic imaging guided photothermal therapy of cancer. Adv Func Mat 25: 1451-1462, 2015

109. Wu M, Guo Q, Xu F, Liu S, Lu X, Wang J, Gao H and Luo P: Engineering phosphopeptide-decorated magnetic nanoparticles as efficient photothermal agents for solid tumor therapy. J Colloid Interface Sci 476: 158-166, 2016.

110. Yu J, Ju Y, Zhao L, Chu X, Yang W, Tian Y, Sheng F, Lin J, Liu F Dong Y and Hou Y: Multistimuli-regulated photochemothermal cancer therapy remotely controlled via Fe5C2 nanoparticles. ACS Nano 10: 159-169, 2016.

111. Zhang M, Cao Y, Wang L, Ma Y, Tu X and Zhang Z: Manganese doped iron oxide theranostic nanoparticles for combined $\mathrm{T}$ magnetic resonance imaging and photothermal therapy. ACS Appl Mat Interfaces 7: 4650-4658, 2015.
112. Guo X, Wu Z, Li W, Wang Z, Li Q, Kong F, Zhang H, Zhu X, Du YP, Jin Y, et al: Appropriate size of magnetic nanoparticles for various bioapplications in cancer diagnostics and therapy. ACS Appl Mater Interfaces 8: 3092-3106, 2016.

113. Bhana S, Lin G, Wang L, Starring H, Mishra SR, Liu G and Huang X: Near-infrared-absorbing gold nanopopcorns with iron oxide cluster core for magnetically amplified photothermal and photodynamic cancer therapy. ACS Appl Mater Interfaces 7: 11637-11647, 2015.

114. Guo R,Peng H,Tian Y, Shen S and Yang W: Mitochondria-targeting magnetic composite nanoparticles for enhanced phototherapy of cancer. Small 12: 4541-4552, 2016.

115. Espinosa A, Di Corato R, Kolosnjaj-Tabi J, Flaud P, Pellegrino T and Wilhelm C: Duality of iron oxide nanoparticles in cancer therapy: Amplification of heating efficiency by magnetic hyperthermia and photothermal bimodal treatment. ACS Nano 10: 2436-2446, 2016 